\title{
A Generic and Flexible Framework for the Geometrical and Topological Analysis of (Algebraic) Surfaces*
}

\author{
Eric Berberich ${ }^{\dagger}$ \\ Max-Planck-Institut für Informatik
}

\author{
Michael Sagraloff ${ }^{\ddagger}$ \\ Max-Planck-Institut für Informatik
}

\begin{abstract}
We present a generic framework on a set of surfaces $\mathcal{S}$ in $\mathbb{R}^{3}$ that provides their geometric and topological analysis in order to support various algorithms and applications in computational geometry. Our implementation follows the generic programming paradigm, i.e., to support a certain family of surfaces, we require a small set of types and some basic operations on them, all collected in a model of the newly presented SURFACETRAITS_3 concept. The framework obtains geometric and topological information on a non-empty set of surfaces in two steps. First, important 0 and 1-dimensional features are projected onto the $x y$-plane, obtaining an arrangement $\mathcal{A}_{\mathcal{S}}$ with certain properties. Second, for each of its components, a sample point is lifted back to $\mathbb{R}^{3}$ while detecting intersections with the given surfaces. This idea is similar to Collins' cylindrical algebraic decomposition (cad). In contrast, we reduce the number of liftings using CGAL's Arrangement_2 package as a basic tool. Properly instantiated, the framework provides main functionality required to support the computation of a Piano Mover's instance. On the other hand, the complexity of the output is high, and thus, we particularly regard the framework as key ingredient for querying information on and constructing geometric objects from a small set of surfaces. Examples are meshing of single surfaces, the computation of space-curves defined by two surfaces, to compute lower envelopes of surfaces, or as a basic step to compute an efficient representation of a three-dimensional arrangement.

We also inspirit the framework in two steps. First, we show that the well-known family of algebraic surfaces fulfils the framework's requirements. As robust implementations on these surfaces are lacking these days, we consider the framework to be an important step to fill this gap. Second, we instantiate the framework by a fully-fledged model for special algebraic surfaces, namely quadrics. This instantiation already supports main tasks demanded from rotational robot motion planning [Latombe 1993]. How to provide a model for algebraic surfaces of arbitrary degree, is partly discussed in [Berberich et al. 2008].
\end{abstract}

CR Categories: J.6 [Computer Applications]: Computeraided Engineering-Computer-aided design (CAD); F.2.2 [Theory of Computation]: Nonnumerical Algorithms and ProblemsGeometrical problems and computations;

Keywords: Surface analysis, geometry, topology, framework, generic programming, meshing, space curves, lower envelopes, algebraic surfaces, quadrics

${ }^{*}$ This work has been supported in part by the IST Programme of the EU as Shared-cost RTD (FET Open) Project under Contract No IST-006413 (ACS - Algorithms for Complex Shapes).

†e-mail: eric@mpi-inf.mpg.de

‡e-mail: msagralo@mpi-inf.mpg.de

This is the authors' version of the work. It is posted here by permission of ACM for your personal use. Not for redistribution. The definitive version was published in the Proceedings of the ACM Solid and Physical Modelling Symposium (SPM 2008) http://doi.acm.org/10.1145/1364901.1364925

\section{Introduction}

Given a finite set $\mathcal{S}=\left\{S_{1}, \ldots, S_{N}\right\}$ of surfaces in $\mathbb{R}^{3}$, we are interested in the geometric and topological information to describe $\mathcal{S}$. So, we aim for a decomposition of $\mathbb{R}^{3}$ with respect to $\mathcal{S}$ into open cells of dimension $0,1,2$, and 3 . While the first three will be computed explicitly, the 3-dimensional cells are interfaced implicitly. We also want to come up with their adjacency relationship and their position in $\mathbb{R}^{3}$. We go into this direction as various algorithms in computational geometry can be expressed in terms of this information.

Our approach uses projection onto the plane, i.e., its basis consists of a finite arrangement $\mathcal{A}_{\mathcal{S}}$, with some invariant properties for all points of a component of $\mathcal{A}_{\mathcal{S}}$. In particular, they share the same $z$-pattern when they are lifted. A $z$-pattern at $p$ encodes the sequence of intersections of $S_{i} \in \mathcal{S}$ with the vertical line $\ell_{p}$ at $p$. It then suffices to compute a $z$-pattern only for a sample point of each component of $\mathcal{A}_{\mathcal{S}}$.

In Section 2 we introduce the main problems that must be tackled and present conditions demanded on a family of surfaces to pursue this strategy. We show that all conditions are fulfilled for algebraic surfaces and give an almost minimal arrangement with the required properties.

Section 3 presents our generic implementation. It is an extension of CGAL's Arrangement_2 package. We introduce a new $\mathrm{C}++$-concept which must be implemented for the favored family of surfaces. The concept abstracts the rather complex challenge into a small set of simple tasks demanded on surfaces, like to compute approximations of $S_{i} \cap \ell_{p}$. It is the accountability of the framework to combine the output of these operations to obtain the desired output. Some examples how to use the framework in interesting applications are discussed in Section 3.4. Section 4 explains how to implement a model of the concept on the example of quadrics, i.e., algebraic surfaces of degree 2 . We conclude with some experimental results.

Related work Our strategy for algebraic surfaces in general follows elimination theory [Basu et al. 2006] and main ideas of cylindrical algebraic decomposition ( $\mathrm{cad}$ ) [Arnon et al. 1984]. A collection of articles emblazing different aspects of cad is given in [Caviness and Johnson 1998]. Many algorithms in computational geometry can be expressed in terms of a cad-instance. A famous example is the Piano Mover's problem that is extensively discussed in [Schwartz et al. 1987]. But unfortunately, many implementations, if any, avoid this technique. We believe for two reasons. One is the quite high complexity of cad. The other is the algebraic focus, that usually requires good knowledge on the topic. Thus, with our framework we want to close the gap, between cad-techniques and implementations of algorithms in computational geometry. Our aim is to provide an easy-to-use framework, with full power on the analysis of surfaces, while always focusing towards applications in computational geometry. As we decouple combinatorics from predicates, it depends on the model used, whether the instantiated framework follows the exact computation paradigm [Yap 1997]. Note that most generic implementations of geometric algorithms show up an undetermined behavior or fail to stop if instantiated with floating-point arithmetic. In the same spirit, we encourage to 
use the framework with exact number types and to apply consistent filters for speed-ups.

Two other fields of research are touched by this work. One is the determination of a surface's topology. Several approaches exist, e.g., [Mourrain and Técourt 2005; Cheng et al. 2005], but they usually assume a generic coordinate system. Recently, an approach without shearing has been proposed in [Berberich et al. 2008]. Algebraic space curves are discussed in [Gatellier et al. 2005].

The other challenging task is the computation of arrangements of several surfaces. Until now, no complete implementation is available. [Mourrain et al. 2005] presented a method to compute arrangements of quadrics using a space-sweep. An implementation is missing. For two quadrics, a specialized projection approach is available as software [Berberich et al. 2005b]. In contrast to that work, the proposed framework can deal with more than two quadrics, allows more surfaces, and does not pose any generic position assumptions. Thus, it can be interpreted as a key step towards arrangements of surfaces.

\section{Problem}

Let $\mathcal{S}=\left\{S_{1}, \ldots, S_{N}\right\}$ be a finite set of surfaces in $\mathbb{R}^{3}$. Throughout this section, we detect some conditions that clarify what we consider to be valid surfaces usable in our framework.

For $p=\left(p_{x}, p_{y}\right)$ a planar point in $\mathbb{R}^{2}$, we denote by $\ell_{p} \subset \mathbb{R}^{3}$ the vertical line through $p$. We tackle the following abstract problems from computational geometry. Abstract means to consider first a surface as set of points.

Problem 2.1. Given a set of surfaces $\mathcal{S}$, compute for an arbitrary point $p \in \mathbb{R}^{2}$ the ordered sequence of intersections of all $S_{i} \in \mathcal{S}$ with $\ell_{p}$.

In order to encode the sequence of intersections of $S_{i} \in \mathcal{S}, i=$ $1, \ldots, N$, with $\ell_{p}$ we use the following definition of a z-pattern.

Definition 2.2 (z-pattern). We call the sequence $G_{\mathcal{S}, p}=g_{1}, \ldots, g_{k}$ of subsets of $\{1, \ldots, N\}$ a $z$-pattern with respect to $\mathcal{S}$ and $p$.

If we fix $p$, Problem 2.1 can be split into two, the consecutive Problems 2.3 and 2.7.

Problem 2.3. Given a surface $S$ compute all intersections of $S$ with $\ell_{p}$.

We denote $V L_{i}:=\left\{p \in \mathbb{R}^{2} \mid \ell_{p} \subset S_{i}\right\}$ the set of all points $p \in \mathbb{R}^{2}$ where $S_{i}$ contains the vertical line $\ell_{p}$.

Condition 2.4. For a given surface $S_{i}$ it holds $\left|V L_{i}\right|$ is finite.

We introduce the following container.

Definition 2.5 (z-stack). Let $S_{i} \in \mathcal{S}, p=\left(p_{x}, p_{y}\right) \in \mathbb{R}^{2}$. A finite subset $Z_{p, i} \subset\left\{z \in \mathbb{R} \mid\left(p_{x}, p_{y}, z\right) \in S_{i}\right\} \cup\{ \pm \infty\}$ is called $\mathrm{z}-$ stack of $S_{i}$ at $p$ if $Z_{p, i}$ contains $\pm \infty$ and $Z_{p, i}=\left\{\ell_{p} \cap S_{i}\right\} \cup\{ \pm \infty\}$ for $p \notin V L_{i}$. We sort its elements in the following way: $-\infty=$ $z_{p, i,-1}<z_{p, i, 0}<\ldots<z_{p, i, m_{p, i}-1}<z_{p, i, m_{p, i}}=+\infty$.

Whereas the container encodes the intersections of a surface $S_{i}$ with $\ell_{p}$ for $p \notin V L_{i}$ it is intended to store interesting $\mathrm{z}$-coordinates of a surface at a given $p \in V L_{i}$. Its actual content with respect to $p \in V L_{i}$ is given by the conditions 2.6 and 2.11 that define how surfaces are allowed to be connected.

We concentrate on the fact, that $m_{p, i}$ denotes its number of finite elements. Obviously, we cannot compute $Z_{p, i}$ for all $p \in \mathbb{R}^{2}$. Thus, we aim for a subdivision of the plane into finitely many connected cells of dimension 0,1 , and 2 with the property that all points of a cell carry the same $m$-value. Such a subdivision can be represented as an arrangement, where cells are called vertices (0), edges (1), and faces (2). An arrangement is induced by continuous curves and isolated points, which already define the vertices and edges. Faces are then implicitly defined. More detailed, we aim for surfaces to fullfill the following condition.

Condition 2.6. Given a surface $S_{i} \in \mathcal{S}$. Then an arrangement $\mathcal{A}_{\left\{S_{i}\right\}}$ with the following properties exists. $\mathcal{A}_{\left\{S_{i}\right\}}$ consists of a finite number of cells and is induced by a finite number of curves and $a$ finite number of isolated points. $\mathcal{A}_{\left\{S_{i}\right\}}$ contains every point in $V L_{i}$ as vertex and each cell $\Gamma$ of $\mathcal{A}_{\left\{S_{i}\right\}}$ is invariant with respect to $m$, i.e., $\forall p_{1}, p_{2} \in \Gamma: m_{p_{1}, i}=m_{p_{2}, i}=: m_{\Gamma, i}$.

As a consequence, it suffices to only consider a sample point $p_{\Gamma}$ of a cell, if one is interested in $m_{p, i}$ for any point of $\Gamma$. The container $Z_{p, i}$ also gives geometric information about $S_{i}$ at $p$. Let $Z_{\Gamma, i}$ denote the z-stack of an arbitrary sample point $p_{0}$ of $\Gamma$.

Before we turn to look at how $\mathrm{z}$-stacks are connected we first consider pairs of surfaces. A remaining task to solve Problem 2.1 is the following problem.

Problem 2.7 (Compare entries of z-stacks). Let $S_{i}, S_{j} \in \mathcal{S}, i \neq j$. Given a point $p$ and $z_{p, i, u} \in Z_{p, i}$ and $z_{p, j, v} \in Z_{p, j}$ decide whether $z_{p, i, u}<z_{p, j, v}, z_{p, i, u}=z_{p, j, v}$, or $z_{p, i, u}>z_{p, j, v}$.

It is easy to see that the application of a divide-and-conquer strategy that combines Problem 2.3 and 2.7 results in a solution of Problem 2.1. We learn in Section 3.1 that the equality decision is sufficient, as we represent a $z_{p, i, u} \in Z_{p, i}$ with a refineable interval approximation. In the spirit of this problem we define another value.

Definition $2.8\left(m_{p, i, j}\right)$. Let $S_{i}, S_{j} \in \mathcal{S}, i \neq j$ and let $p \in \mathbb{R}^{2}$. Then $m_{p, i, j}:=\left|\left(Z_{p, i} \cap Z_{p, j}\right) \backslash\{ \pm \infty\}\right|$. For an $m_{p, i, j}$ - invariant connected set $\Gamma$ we define $m_{\Gamma, i, j}:=m_{p, i, j}$ with a $p \in \Gamma$.

Again, we can neither compute the intersections of z-stacks nor $m_{p, i, j}$ for an infinite number of points. This fact founds another condition on the surfaces.

Condition 2.9. Given surfaces $S_{i}, S_{j} \in \mathcal{S}, i \neq j$. Then an arrangement $\mathcal{A}_{\left\{S_{i}, S_{j}\right\}}$ exists, with: $\mathcal{A}_{\left\{S_{i}, S_{j}\right\}}$ consists of a finite number of cells and is induced by a finite number of curves and $a$ finite number of isolated points. $A_{i, j}$ contains every point of $V L_{i} \cup V L_{j}$ as a vertex and for each cell $\Gamma$ of $\mathcal{A}_{\left\{S_{i}, S_{j}\right\}}$, the following equations hold: $\forall p_{1}, p_{2} \in \Gamma: m_{p_{1}, i}=m_{p_{2}, i}, m_{p_{1}, j}=m_{p_{2}, j}, m_{p_{1}, i, j}=$ $m_{p_{2}, i, j}$.

Problem 2.10 (Compute planar arrangements). For given surfaces $S_{i}, S_{j} \in \mathcal{S}, i \neq j$, compute $\mathcal{A}_{\left\{S_{i}\right\}}, \mathcal{A}_{\left\{S_{j}\right\}}$, and $\mathcal{A}_{\left\{S_{i}, S_{j}\right\}}$.

It is missing, which connectivity between $Z_{\Gamma_{1}, i}$ and $Z_{\Gamma_{2}, i}$, for $\Gamma_{1}, \Gamma_{2}$ being incident cells of $\mathcal{A}_{\left\{S_{i}\right\}}$, is allowed:

Condition 2.11. Let $S_{i} \in \mathcal{S}$, and $\mathcal{A}_{\left\{S_{i}\right\}}$ an m-regular arrangement. Then $S_{i}$ is continuous in the following sense:

1. Let $p_{n} \in \Gamma \in \mathcal{A}_{\left\{S_{i}\right\}}$ be a sequence of points with $\lim _{n \rightarrow \infty} p_{n}=p \in \Gamma^{*} \in \mathcal{A}_{\left\{S_{i}\right\}}$, and $Z_{p_{n}, i}=$ $\left\{z_{p_{n},-1}, \ldots, z_{p_{n}, m_{i, \Gamma}}\right\}$. Then for any $k \in\left\{-1,0, \ldots, m_{i, \Gamma}\right\}$ we have $\left\{\lim _{n \rightarrow \infty} z_{p_{n}, k}: p_{n} \in \Gamma\right.$ with $\left.p_{n} \rightarrow p\right\}=$ $\left[z_{p, v_{k}^{-}}, z_{p, v_{k}^{+}}\right]$with $z_{p, v_{k}^{ \pm}} \in Z_{p, i}$ and $\lim _{n \rightarrow \infty} z_{p_{n}, k} \leq$ $\lim _{n \rightarrow \infty} z_{p_{n}, l}$ for $k<l$.

2. For $p \notin V L_{i}$ each interval $\left[z_{p, v_{k}^{-}}, z_{p, v_{k}^{+}}\right]$consists of exactly one point, i.e., $z_{p, v_{k}^{-}}=z_{p, v_{k}^{+}}$.

This neighborhood-relationship suffices to encode the connectivity of all lifted cells. We remark that from the above conditions it follows that each cell $\Gamma \in \mathcal{A}_{\left\{S_{i}\right\}} \backslash V L_{i}$ is the projection of $m_{\Gamma, i}-2$ connected, disjoint cells of $S_{i}$ respectively. The lifts of these cells 
together with the decomposition of vertical lines $\ell_{p}$ into subsegements $\left(p,\left[z_{p, v_{k}^{-}}, z_{p, v_{k}^{+}}\right]\right)$form a decomposition of $S_{i}$ which has the boundary property, i.e., the boundary of a cell is given by a union of other cells (compare the similar notion of a CW-complex [Massey 1967], [Bredon 1993]). An analogous formulation also holds for the arrangement $A_{i, j}$ with respect to the surfaces $S_{i}, S_{j}$ and their intersection $S_{i} \cap S_{j}$.

Whereas the z-stack at a point $p \notin V L_{i}$ was uniquely determined, its content at points $p \in V L_{i}$ is only implicitely (not uniquely) given by the chosen arrangement and Condition 2.11.

Problem 2.12 (Compute z-stack). For given surface $S_{i} \in \mathcal{S}$ and given point $p \in \mathbb{R}^{2}$, compute $Z_{p, i}$, even if $p \in V L_{i}$.

It remains to compute the connections between lifted cells which is encoded in terms of connections between lifted sample points.

Problem 2.13 (Adjacency). Given $\mathcal{A}_{\left\{S_{i}\right\}}$ for a surface $S_{i} \in \mathcal{S}$. Let $\Gamma_{1}, \Gamma_{2}$ denote incident cells of $\mathcal{A}_{\left\{S_{i}\right\}}$ and $p_{1}, p_{2}$ their respective planar sample points. Then we are interested in how an entry of $Z_{1}:=Z_{\Gamma_{1}, i}$ is connected with the intervals defined by the entries of $Z_{2}:=Z_{\Gamma_{2}, i}$. We are asking for a list $L$ of pairs $(a, b) \in A \times B$, with $A:=\left\{0, \ldots, m_{\Gamma_{1}, 1}-1\right\}$ and $B:=\left\{-1, \ldots, m_{\Gamma_{2}, 1}\right\}$. We distinguish 5 cases for a fixed $a_{0}$ :

$\left\{b:\left(a_{0}, b\right) \in L\right\}=\emptyset:$ Indicates, that there exists no continuous path on $S_{i}$ whose closure connects $\left(p_{1}, z_{p_{1}, i, a_{0}}\right)$ with some $\left(p_{2}, z_{p_{2}, i, b}\right), b \in B$.

$\left\{b:\left(a_{0}, b\right) \in L\right\}=\left\{b_{0}\right\} \wedge b_{0} \notin\left\{-1, m_{\Gamma_{2}, i}\right\}:$ The pair $\left(a_{0}, b_{0}\right)$ then denotes the existence of a continuous path on $S_{i}$, lying over $\Gamma_{1}$, whose closure connects $\left(p_{1}, z_{p_{1}, i, a_{0}}\right)$ with $\left(p_{2}, z_{p_{2}, i, b_{0}}\right)$.

$\left\{b:\left(a_{0}, b\right) \in L\right\}=\left\{b_{0}\right\} \wedge b_{0}=-1\left(b_{0}=m_{\Gamma_{2}, i}\right):$ The pair $\left(a_{0}, b_{0}\right)$ denotes the existence of a continuous path, lying over $\Gamma_{1}$, whose closure connects $\left(p_{1}, z_{p_{1}, i, a_{0}}\right)$ with the infinite "point" $\left(p_{2},-\infty\right)\left(\left(p_{2},+\infty\right)\right)$, i.e., $S_{i}$ has a vertical asymptote with respect to $z$ at $p_{2}$.

$\left|\left\{\left(a_{0}, b\right) \in L\right\}\right|=2$ : Let $\left(a_{0}, b_{0}\right)$ and $\left(a_{0}, b_{1}\right)$ be these pairs. They denote the existence of an infinite number of continuous paths on $S_{i}$, lying over $\Gamma_{1}$, such that exactly all points $\left(p_{2}, z\right), z \in\left[z_{p_{2}, i, b_{0}}, z_{p_{2}, i, b_{0}}\right]$ are connected with $\left(p_{1}, z_{p_{1}, i, a_{0}}\right)$ by considering the closure of a path. Only in case that $b_{0}=-1$ or $b_{1}=m_{\Gamma_{2}, i}$, the interval is meant to be open at that end.

Note that we only compute adjacencies between 0-,1-, and 2dimensional cells. The adjacencies to 3-dimensional open cells are given implicitly by them and the projection technique.

We can state our final problem.

Problem 2.14. Given a set of surfaces $\mathcal{S}$ fulfilling Conditions 2.4, 2.6, 2.9, and 2.11. Compute a finite planar arrangement $\mathcal{A}_{\mathcal{S}}$ with the property that for each of its cells $\Gamma$ it holds: $\forall p_{1}, p_{2} \in \Gamma$ : $G_{\mathcal{S}, p_{1}}=G_{\mathcal{S}, p_{2}}$. In addition, we want to know how the entries of z-patterns of incident cells in $\mathcal{A}_{\mathcal{S}}$ are connected.

We claim that our framework implements such a solution for Problem 2.14 given the fact that proper implementations for Problems 2.10 (planar arrangements), 2.12 (z-stacks), 2.7 (compare entries of z-stacks), and 2.13 (adjacency) are provided. The last can be used to derive the desired connectivity of z-pattern entries from the connectivity of z-stacks of single surfaces. In terms of implementation details, we already remark, that implementations for these simpler problems are expected as part of a model that fulfills the
SURFACETRAITS_3 concept. We are newly introducing this concept with all its details in Section 3.1.

We show in Section 2.1 for algebraic surfaces, how to obtain $\mathcal{A}_{\left\{S_{i}\right\}}$ and $\mathcal{A}_{\left\{S_{i}, S_{j}\right\}}$ without actually computing any z-stack. That section also shows that irreducible algebraic surfaces, our motivating example for the framework, fulfill all stated conditions.

\subsection{Algebraic Surfaces}

Definition 2.15 (Algebraic surface). An irreducible algebraic surface $S \subset \mathbb{R}^{3}$ is defined by the real valued vanishing set of an irreducible polynomial $f=a_{n}(x, y) z^{n}+\ldots+a_{0}(x, y) \in \mathbb{R}[x, y, z]$.

Because of our assumption the polynomial coefficients $a_{i}(x, y)$ do not share a common component, thus their corresponding planar curves $A_{i}:=V\left(a_{i}\right)$ either intersect in finitely many points or $f=$ $a_{0}(x, y)$. This follows from Bézout's Theorem which says that two planar algebraic curves of degree $d$ and $e$, that do not share a common component, intersect in at most $d \cdot e$ points. Thus the number of common intersection points of $A_{i}$ is finite, too. It holds for a finite set of irreducible, distinct algebraic surfaces that $\operatorname{dim}\left(S_{i} \cap S_{j}\right)<2$, which is a direct consequence of the irreducibility of each surface as two surfaces cannot contain a 2-dimensional component in this case. Under the assumption that Condition 2.9 holds, for $p \notin V L_{i}$, Condition 2.11 can be verified by the fact that the roots of a polynomial continuously depend on its coefficients, i.e., we must get $\left\{\lim _{n \rightarrow \infty} z_{p_{n}, i,-1}, \ldots, \lim _{n \rightarrow \infty} z_{p_{n}, i, m_{i, \Gamma}}\right\} \subset Z_{p, i}$ and $\lim _{n \rightarrow \infty} z_{p_{n}, i, k} \leq \lim _{n \rightarrow \infty} z_{p_{n}, i, l}$ for $k<l$. For $p \in V L_{i}$ Theorem 2.21 in combination with Definition 2.22 will show that Condition 2.11 also holds in this case.

Now we provide the following (constructive) definition of $\mathcal{A}_{\mathcal{S}}$ :

We do not only prove the existence of an arrangement with the desired properties but we will also aim for constructing such an arrangement which is almost minimal with respect to the number of faces, edges and vertices. In order to detect points where the $z$ pattern changes we need some terms. For two given polynomials $f, g \in \mathbb{R}[x, y, z]$, let $\mathrm{SR}_{i_{0}}(f, g, z) \in \mathbb{R}[x, y]$ denote their subresultant polynomial and $\operatorname{sr}_{i_{0}}(f, g, z)$ its leading coefficient (with respect to $z$ ). For the exact definitions, as well as for the proof of the Theorem 2.16 we refer to [Basu et al. 2006].

Theorem 2.16. Let $\mathbb{D}$ be a domain and $f, g \in \mathbb{D}[z]$ be polynomials of degree $m$ and $n$ respectively and $i_{0}:=\min \left\{i \mid \operatorname{sr}_{i}(f, g, z) \neq\right.$ $0\}$. Then $\operatorname{gcd}(f, g)=\mathrm{SR}_{i_{0}}(f, g, z)$ and $\operatorname{deg} \operatorname{gcd}(f, g)=i_{0}$. Especially for $g=f_{z}=\frac{\partial}{\partial z} f$ it follows that $f$ has a multiple (complex) root if and only if $\operatorname{sr}_{0}\left(f, f_{z}, z\right)=0$.

We remark that $\mathrm{SR}_{i_{0}}(f, g, z)$ can be obtained as determinant of a Sylvester submatrices. Its entries are expressions in terms of the coefficients of $f \in \mathbb{R}[x, y][z]$. The definition of $\operatorname{SR}_{i}(f, g, z)$ depends on the degree of $f$ and $g$ with respect to $z$. For this reason we first have to decompose the plane into components $\Gamma$ such that for each point $p \in \Gamma$ the polynomial $f(p, z)$ has the same degree. We introduce the following notation.

Definition 2.17. Let $S=V(f) \subset \mathbb{R}^{3}$ be a surface of degree $n$ with respect to $z$. Then we call a point $p=\left(p_{x}, p_{y}\right) \in \mathbb{R}^{2} m$-regular if and only if $m=\max \left\{i: a_{i}\left(p_{x}, p_{y}\right) \neq 0\right\}$. If $a_{i}\left(p_{x}, p_{y}\right)=0$ for all $i$, then $S$ contains the vertical line $\ell_{p}$. In this situation we call $p$ $(-1)$-regular.

As already mentioned we aim for a decomposition of $\mathbb{R}^{2}$ in terms of regularity. This can be achieved by considering the arrangement induced by the planar curves $A_{i}$, i.e., the vanishing sets of bivariate polynomials $a_{i}$. This arrangement is a decomposition of $\mathbb{R}^{2}$ into faces, consisting of $n$-regular points, and edges and vertices, consisting of points of lower regularity. Notice that it is not necessary to consider the entire arrangement of all $A_{i}$ : Starting with the arrangement $\mathcal{A}_{S}^{(0)}$ induced by $A_{n}$ one 
can iteratively define $\mathcal{A}_{S}^{(j)}$ as the refined arrangement of $\mathcal{A}_{S}^{(j-1)}$ by inserting the (local 0 -dimensional) intersection points of edges $E \subset A_{n} \cap \ldots \cap A_{n-j+1}$ in $\mathcal{A}_{S}^{(j-1)}$ with $A_{n-j}$. According to this construction in each step exactly those components are added which are locally extremal with respect to regularity. Thus we finally end up with an arrangement $\mathcal{A}_{\{S\}}^{*}$ where for each component a certain regularity is allocated. As the $S_{i}$ do not contain two-dimensional vertical components, all $A_{i}$ in at most finitely many points, that define $V L_{i}$. This shows that Condition 2.4 is fulfilled, too.

For a collection $\mathcal{S}:=\left\{S_{1}, \ldots, S_{N}\right\}$ of algebraic surfaces $S_{i}=$ $V\left(f_{i}\right)=V\left(a_{i, n_{i}}(x, y) z^{n_{i}}+\ldots+a_{i, 0}(x, y)\right)$ of degree $n_{i}$ with respect to $z$, Definition 2.17 has a natural extension.

Definition 2.18. We call a point $p=\left(p_{x}, p_{y}\right) \in \mathbb{R}^{2}$ $\left(m_{1}, \ldots, m_{N}\right)-$ regular with respect to $\mathcal{S}:=\left\{S_{1}, \ldots, S_{N}\right\}$ if and only if $p$ is $m_{i}$-regular with respect to $S_{i}$.

Let $S_{1}, S_{2} \in \mathcal{S}$ be two surfaces and $\mathcal{A}_{\left\{S_{1}, S_{2}\right\}}^{*}$ the overlay of the arrangements $\mathcal{A}_{S_{1}}^{*}$ and $\mathcal{A}_{S_{2}}^{*}$, then for each component of $\mathcal{A}_{\left\{S_{1}, S_{2}\right\}}^{*}$ the regularity with respect to $\left\{S_{1}, S_{2}\right\}$ stays invariant. So for each component we can use Theorem 2.16 to get information about the $z$-patterns $G_{\left\{S_{j}\right\}, p}, j=1,2$ and $G_{\left\{S_{1}, S_{2}\right\}, p}$. We will show that there exists a refinement $\mathcal{A}_{\left\{S_{1}, S_{2}\right\}}$ of $\mathcal{A}_{\left\{S_{1}, S_{2}\right\}}^{*}$, such that on each component $\Gamma \in \mathcal{A}_{\left\{S_{1}, S_{2}\right\}}$ the $z$-patterns $G_{\left\{S_{j}\right\}, p}, j=1,2$, and $G_{\left\{S_{1}, S_{2}\right\}, p}$ stay the same. For this purpose we have to introduce some further notation.

Definition 2.19. Let $p=\left(p_{x}, p_{y}\right)$ be an $\left(m_{1}, m_{2}\right)$-regular point. Then $p$ has degradation $i_{0}$ with respect to $\left\{S_{j}\right\}$ iff $i_{0}=$ $\min \left\{i \mid \operatorname{sr}_{i}\left(f_{j}\left(p_{x}, p_{y}, z\right), f_{j_{z}}\left(p_{x}, p_{y}, z\right), z\right) \neq 0\right\}$. We say that $p$ has degradation $i_{12}$ with respect to $\left\{S_{1}, S_{2}\right\}$ iff $i_{12}:=\min \{i \mid$ $\left.\operatorname{sr}_{i}\left(f_{1}\left(p_{x}, p_{y}, z\right), f_{2}\left(p_{x}, p_{y}, z\right), z\right) \neq 0\right\}$.

Let $\Gamma \in \mathcal{A}_{\left\{S_{1}, S_{2}\right\}}^{*}$ be a component of regularity $\left(m_{1}, m_{2}\right)$, then there exist common minimal degradations $i_{j, \Gamma}$ and $i_{12, \Gamma}$ for all points on $\Gamma$ with respect to $\left\{S_{j}\right\}$ and $\left\{S_{1}, S_{2}\right\}$. More precisely $i_{j, \Gamma}=\min _{p \in \Gamma} \min \left\{i \mid \operatorname{sr}_{i}\left(f_{j}(p, z), f_{j}(p, z), z\right) \neq 0\right\}$ and $i_{12, \Gamma}=\min _{p \in \Gamma} \min \left\{i \mid \operatorname{sr}_{i}\left(f_{1}(p, z), f_{2}(p, z), z\right) \neq 0\right\}$.

For a face $\Gamma \in \mathcal{A}_{\left\{S_{1}, S_{2}\right\}}^{*}$ all points on $\Gamma$ have degradations $i_{j, \Gamma}=$ $i_{12, \Gamma}=0$, except those where $\operatorname{sr}_{0}\left(f_{j}, f_{j_{z}}, z\right)$ or $\operatorname{sr}_{0}\left(f_{1}, f_{2}, z\right)$ vanishes. We denote $\Sigma_{j}:=V\left(\operatorname{sr}_{0}\left(f_{j}, f_{j}, z\right)\right)$, the projected silhouette-curve of $S_{j}$ and $\Sigma_{1,2}:=V\left(\operatorname{sr}_{0}\left(f_{1}, f_{2}, z\right)\right)$ the projected cut-curve of $S_{1}$ and $S_{2}$. Now consider the overlay $\mathcal{A}_{\left\{S_{1}, S_{2}\right\}}^{* *}$ of $\mathcal{A}_{\left\{S_{1}, S_{2}\right\}}^{*}$ and the planar curves $\Sigma_{j}$ and $\Sigma_{1,2}$. Every face of $\mathcal{A}_{\left\{S_{1}, S_{2}\right\}}^{* *}$ consists of points $p$ with the same $z$-patterns $G_{\left\{S_{j}\right\}, p}$ and $G_{\left\{S_{1}, S_{2}\right\}, p}$ as a change in one of these patterns can only occur in case of a multiple root of $f_{j}(p, z)$ or in case of a common root of $f_{1}(p, z)$ and $f_{2}(p, z)$.

It remains to refine the edges of $\mathcal{A}_{\left\{S_{1}, S_{2}\right\}}^{* *}$ to get an arrangement with the desired properties. Let $E \in \mathcal{A}_{\left\{S_{1}, S_{2}\right\}}^{* *}$ be an $\left(m_{1}, m_{2}\right)$-regular edge with minimal common degradations $i_{j, E}$ and $i_{12, E}$ with respect to $\left\{S_{j}\right\}$ and $\left\{S_{1}, S_{2}\right\}$. Now passing $E$, a change in one of the patterns $G_{\left\{S_{j}\right\}, p}$ or $G_{\left\{S_{1}, S_{2}\right\}, p}$ can only occur at points $p \in E$ where $\operatorname{sr}_{i_{j}, E}\left(f_{j}, f_{j_{z}}, z\right)$ or $\operatorname{sr}_{i_{12, E}}\left(f_{1}, f_{2}, z\right)$ vanishes. The intersection points of these algebraic curves with $E$ are saved as vertices in the arrangement, which leads to a refined arrangement $\mathcal{A}_{\left\{S_{1}, S_{2}\right\}}$ of $\mathcal{A}_{\left\{S_{1}, S_{2}\right\}}^{* *}$ that has properties as required in Condition 2.9. The construction also shows that we can attach a degradation $i_{j, \Gamma}$ and $i_{12, \Gamma}$ for each component $\Gamma$ of $\mathcal{A}_{\left\{S_{1}, S_{2}\right\}}$ with respect to $\left\{S_{j}\right\}$ and $\left\{S_{1}, S_{2}\right\}$.

Observe that by construction every edge $E \in \mathcal{A}_{\left\{S_{1}, S_{2}\right\}}$ is completely contained in one of the curves $V\left(a_{j, n_{j}}\right), \Sigma_{j}$ or $\Sigma_{1,2}$. However, $\operatorname{sr}_{0}\left(f_{j}, f_{j}, z\right)$ contains a factor $a_{j, n_{j}}$, thus $\Sigma_{j}$ contains $V\left(a_{j, n_{j}}\right)$. It follows that the arrangement $\mathcal{A}_{\Sigma_{j}, \Sigma_{1,2}}$ which is induced by $\Sigma_{j}$ and $\Sigma_{1,2}$ already defines the faces of $\mathcal{A}_{\left\{S_{1}, S_{2}\right\}}$. In order to obtain $\mathcal{A}_{\left\{S_{1}, S_{2}\right\}}$ the edges of $\mathcal{A}_{\Sigma_{j}, \Sigma_{1,2}}$ have to be refined by adding vertices which result from intersection with coefficient polynomials $a_{i}$ and higher order subresultants $\operatorname{sr}_{i}$ as shown above.

Definition 2.20. Let $\mathcal{S}=\left\{S_{1}, \ldots, S_{N}\right\}$ and $\mathcal{A}_{\left\{S_{i}, S_{j}\right\}}$ the arrangement as constructed above. Then we define $\mathcal{A}_{\mathcal{S}}$ to be the overlay of all arrangements $\mathcal{A}_{\left\{S_{i}, S_{j}\right\}}$.

From our definition, $\mathcal{A}_{\mathcal{S}}$ consists of components $\Gamma \in \mathcal{A}_{\mathcal{S}}$ such that $G_{\mathcal{S}, p}$ is identical for all $p \in \Gamma$. It remains to give an exact definition of $Z_{p, i}$ for a surface $S_{i} \in \mathcal{S}$, which contains the vertical line $\ell_{p}$, and to show that Condition 2.11 is fulfilled in this situation, too. We remark that $p$ is a vertex in $\mathcal{A}_{S}$ and for a neighborhood of $p$, none of the surfaces $S_{j}$ contains a vertical line, except at $p$. Now we consider a sequence of points $p_{n} \in \Gamma \subset \mathcal{A}_{\mathcal{S}}$ that converge against $p$. Then we have to determine possible limits of their $k$-th lifts $\left(p_{n}, z_{p_{n}, i, k}\right) \subset p_{n} \times Z_{p_{n}, i} \subset S_{i}$ with respect to $S_{i}$. If all $p_{n}$ lie on an edge $E$, then the limit is uniquely given as endpoint (above $p$ ) of the $k$-th lift of $E$ with respect to $S_{i}$. For a face $F \in \mathcal{A}_{\mathcal{S}}$, adjacent to $p$, it can happen that the limits of the $k$-th lifts of two different sequences $p_{n}, p_{n}^{\prime} \in F$ are distinct.

Theorem 2.21. Given a surface $S_{i} \in \mathcal{S}, \Gamma \in \mathcal{A}_{\mathcal{S}}$ and two sequences $p_{n}, p_{n}^{\prime} \in \Gamma$ with $p=\lim _{n \rightarrow \infty} p_{n}^{\prime}=\lim _{n \rightarrow \infty} p_{n}$ and $z_{0}=\lim _{n \rightarrow \infty} z_{p_{n}, i, k}, z_{1}:=z_{p_{n}^{\prime}, i, k}$, then for any $z^{*}$ in between $z_{0}$ and $z_{1}$ there exists a sequence $p_{n}^{*} \in \Gamma$ with $p=\lim _{n \rightarrow \infty} p_{n}^{*}$ and $z^{*}=\lim _{n \rightarrow \infty} z_{p_{n}^{*}, i, k}$.

Proof. If $z_{0}=z_{1}$ there is nothing to prove, thus we can assume $z_{0}<z_{1}$. We can further assume that $\left|p_{n}-p\right|$ and $\left|p_{n}^{\prime}-p\right|$ are monotone. From the definition of $\mathcal{A}_{S}$ it follows that there exists an $\epsilon_{0}$ such that $U_{\epsilon} \cap \Gamma$ is connected for all $\epsilon<\epsilon_{0}$ and $U_{\epsilon}:=\left\{q \in \mathbb{R}^{2}\right.$ : $|q-p| \leq \epsilon\}$. Thus we can assume that $U_{n} \cap \Gamma$, where $U_{n}:=\{q \epsilon$ $\left.\mathbb{R}^{2}|| q-p \mid \leq 2 \max \left\{\left|p_{n}-p\right|,\left|p_{n}^{\prime}-p\right|\right\}\right\}$, is connected. Now we consider a continuous path $\Pi_{n} \subset \Gamma \cap U_{n}$, that connects $p_{n}$ and $p_{n}^{\prime}$. As the roots of $f(q, z)$ continuously depend on the point $q \in \Gamma$, for each $z_{n}^{*}$ in between $z_{p_{n}, i, k}$ and $z_{p_{n}^{\prime}, i, k}$ we can choose a $p_{n}^{*}$ that lifts to $z_{p_{n}^{*}, i, k}=z_{n}^{*}$. As $z_{0}=\lim _{n \rightarrow \infty} z_{p_{n}, i, k}$ and $z_{1}:=z_{p_{n}^{\prime}, i, k}$ there exists an $n_{0} \in \mathbb{N}$ such that $z^{*} \in\left[z_{p_{n}, i, k}, z_{p_{n}^{\prime}, i, k}\right]$ for all $n>n_{0}$. Thus we can choose $z_{n}^{*}=z^{*}$ from which it follows that $p_{n}^{*} \in \Gamma$ converges against $p$ and fulfills $z^{*}=\lim _{n \rightarrow \infty} z_{p_{n}^{*}, i, k}$.

Theorem 2.21 shows that for any element $\Gamma \in \mathcal{A}_{\mathcal{S}}$, adjacent to $p$, and any $k \in\left\{-1, \ldots, m_{i, \Gamma}\right\}$, the set of $\operatorname{limits}_{\lim } \lim _{n \rightarrow \infty} z_{p_{n}, i, k}$ $\left(p_{n} \in \Gamma\right.$ a sequence that converge against $\left.p\right)$ is an interval of $I_{\Gamma, i, k} \subset \mathbb{R}$. Thus defining $Z_{p, i}$ to be the set of all endpoints of these intervals, fulfills Condition 2.11.

Definition 2.22 ( $z$-stack). Let $\mathcal{S}=\left\{S_{1}, \ldots, S_{N}\right\}$ be a set of irreducible algebraic surface and $\mathcal{A}_{\mathcal{S}}$ as defined above. For a surface $S_{i} \in \mathcal{S}$ the $z$-stack $Z_{p, i}$ is defined as follows:

- $Z_{p, i}:=\left\{z \in \mathbb{R} \mid\left(p_{x}, p_{y}, z\right) \in S_{i}\right\} \cup\{ \pm \infty\}$ for $p \notin V L_{i}$

- $Z_{p, i}:=\left\{z \in \mathbb{R} \mid \exists \Gamma \in \mathcal{A}_{\mathcal{S}}, k \in\left\{-1, \ldots, m_{i, \Gamma}\right\}\right.$ such that $z$ is an endpoint of $\left.I_{\Gamma, i, k}\right\}$ for $p \in V L_{i}$

\section{Framework}

This section reports on details about the implementation of the framework. The algorithmic basis consists of planar arrangements, thus we rely on the matured Arrangement_2 package [Wein et al. 2007a] of CGAL, the Computational Geometry Algorithm Library. ${ }^{1}$ The version shipped with CGAL 3.4 supports several unbounded faces [Berberich et al. 2007]. We omit to repeat how to construct and maintain an arrangement and refer

${ }^{1}$ See project homepage: www. cgal .org 
to [Agarwal and Sharir 2000] and CGAL's manual pages. Anyhow, we highlight parts relevant for our purpose. As throughout CGAL, the package implements the generic programming paradigm [Austern 1999], i.e., its actual behavior is determined at compile-time by instantiating template parameters. The important template parameter for our purpose is the GEOMETRYTRAITS that has to fulfill CGAL's ARRANGEMENTTRAITS 2 concept. The concept defines three types: Curve_2 that are not necessarily $x$ monotone, $\mathrm{X} \_$monotone_curve_2 to represent $x$-monotone subcurves, and Point_2 to represent finite endpoints of curves, isolated points, and intersections points. It also defines operations on these types, e.g., to split an instance of type Curve_2 into X_monotone_curve_2s and its isolated points. Other operations are used to construct, maintain, and query arrangements of them. We refer to CGAL's manual pages for more details on this. The Arrangement_2 package also provides an incremental construction method (zone-computation) and offers the well-known sweep line paradigm [Bentley and Ottmann 1979] for aggregated constructions as well. An arrangement is maintained as a doubly-connectededge-list (DCEL) with sophisticated traversals for its components, i.e., vertices, edges, and faces. The package also supports efficient point-location strategies.

Two tools are very important for us. First, it is possible to extend each DCEL-object with a data type. Section 3.2 presents the attached data required for our framework. Second, efficient overlay of arrangements is implemented using the sweep line paradigm, even if the input arrangements are extended by data. A model of the concept OverlayTraits has to take care of how to merge data associated to the DCEL-objects of the two originating arrangements [Wein et al. 2007b].

The framework itself is currently part of the SwEEPX library of EXACUS, that is a collection of $\mathrm{C}++$-libraries for Efficient and Exact Algorithms for Curves and Surfaces [Berberich et al. 2005a]. ${ }^{2}$ Major parts of EXACUS are currently on the way to be merged into CGAL. In that mood, the framework can be seen as a prototypical CGAL-package, that will be fully integrated in a future release of CGAL. All framework-specific code consists of about 10,000 lines, i.e., not counting CGAL's arrangement package or other basic classes of the library. Let us first present in Section 3.1 the full concept that a model has to fulfill to use the framework with a new family of surfaces. Sections 3.2 and Section 3.3 give details on how to maintain and to extend planar arrangements to serve our needs. Finally, Section 3.4 exemplary presents concrete applications that emerge from the framework.

\subsection{SURFACETRAITS_3-concept}

Our framework must be instantiated with a model of the SURFACETRAITS_3 concept, i.e., a class that implements a collection of syntactic and semantic premises. It does not make assumptions on how to implement them, as long as the demanded functionality is ensured and supported by the formal parameters. A valid model is expected to provide three main types.

Surface_3: Used to represent an $S_{i} \in \mathcal{S}$

Arrangement_traits_2: This type is a model of CGAL's ARRANGEMENTTRAITS 2 concept to enable planar arrangements consisting of its X_monotone_curve_2s and isolated Point_2s. We use it to construct $\mathcal{A}_{\left\{S_{i}\right\}}$ and $\mathcal{A}_{\left\{S_{i}, S_{j}\right\}}$ and their overlays. It depends on the family of surfaces which model is sufficient. The embedded type Curve_2 will be used to represent a planar curve, that can be decomposed into 0- and 1-dimensional components.

Z_at_xy_isolator: An instance of this type represents and approximates the set $Z_{p, i} \backslash\{ \pm \infty\}$ for a given surface $S_{i}$ at a given Point_2 $p$ as refineable intervals. The member number_of_z_coordinates() gives their number,

${ }^{2}$ See project homepage: www.mpi-inf.mpg.de/EXACUS i.e., encodes $m_{p, i}$. The values $z=\{ \pm \infty\}$ are implicitly handled. Refineable means that the $z$-coordinate with index $0 \leq i<$ number_of_z_coordinates () might not be known exactly, but at least a lower and an upper boundary is accessible by lower_boundary (int i) and upper_boundary (int i). This approximation can be improved by refine_interval (int i). The type of such a boundary is given by Z_at_xy_isolator: : Boundary.

Besides these types, also some functors are required. We omit to be as precise as a reference manual would, as we want to emphasize the simplicity of the each task

Construct_surface_curves_2: This functor has to provide three operator() $\mathrm{s}$ that compute different planar curves emanating from a given surface $S$. The output is returned as std::pair< Curve_2, unsigned int > through an OutputIterator. The unsigned int defines the multiplicity of a curve, if possible to compute, else -1 is chosen. For example, bivariate polynomials defining algebraic curves can be factorized by multiplicity. The first operator returns all curves that belong to the projection of the silhouette of $S$, i.e., all 0 - and 1-dimensional parts of $S$, where the $z$-pattern for some $p$ changes, when slightly moving $p$ away from the projection. The second computes for given $m$ all curves whose points can decrease the regularity of these planar points to $m$. The last operator compute for given regularity $m$ and given $0 \leq i<m$ all curves whose points can increase a planar point's degradation to $i$ with respect to the given surface.

Construct_surface_pair_curves_2: This functor is very similar to the previous one. It uses the same interface, that here works with respect to two given surfaces surfacel and surface2. Only two operators are demanded from the model. The first operator returns all curves belonging to the projected intersection of the two surfaces. Note that we assumed surfaces to intersect at most 1-dimensional. The second operator returns for given regularity $m_{1,2}=\min \left(m_{1}, m_{2}\right)$ and given $0 \leq i<m_{1,2}$ all curves whose points can increase a planar point's degradation to $i$ with respect to the two surfaces.

Construct_isolator: Constructs for given point and surface_i the correct instance of Z_at_xy_isolator type, i.e., it computes $Z_{p, i}$. The given point is a sample point for a cell $\Gamma$ of $\mathcal{A}_{\{\text {[surface] }\}}$. To trigger a special or more efficient implementation, integral values carrying the point's regularity and degradation (or even multiplicity in case of edge) with respect to the given surfaces are interfaced

Equal_z: Checks whether two given intervals of two isolators at a common point are equal, as even in simple cases this decision cannot be finally deduced by iterated refinements of the isolating intervals. Before calling the functor, a set of filters is applied, see Algorithm 3.2 for details. In particular, we know, when called, that all intervals of the two given isolators are already refined such, that each interval overlaps with at most one interval of the other isolator. Thus, the set of overlaps forms a candidate list of real intersections. The functor has to decide for the queried candidate, whether there is really an intersection or whether the isolating intervals will separate after a finite number of refinements. Again, the point's regularities and degradations (and multiplicities in case of an edge) with respect to the given surfaces and the pair of surfaces are interfaced. This may improve the Equal_z's performance, as it usually implements the costly computations, e.g., unavoidable symbolic evaluations in some cases of algebraic surfaces.

Adjacency: Given a Surface_3 with index $i$, and let $\Gamma_{1}$ and $\Gamma_{2}$ be two given incident cells of $\mathcal{A}_{\left\{S_{i}\right\}}$. For each $\Gamma$ an instance of type Z_at_xy_isolator at respective sample points is also interfaced. The functor is expected to compute the list $L$ as stated in Problem 2.13 represented as std: :list< std: : pair< int, int $>>$

This concludes the discussion of the SURFACE_TRAITS_3 con- 
cept. We strongly encourage to deploy an extensive caching strategy when implementing these functors to avoid unnecessary recomputations.

\subsection{Planar arrangements and attached data}

The central class of our framework is called SoX::Projection_2. It is a reference-counted [Kettner 2006] version of CGAL's Arrangement_2 instantiated with Arrangement_traits_2 and using CGAL's Arr_extended_dcel to attach an internal data class SoX: :P_dcel_data to each DCEL-vertex, each DCELedge, and each DCEL-face. The class maintains maps to involved surfaces and pairs of surfaces, along with their multiplicities, regularities, and degradations as presented in Section 2. We also store maps to access originating DCEL-handles, after arrangements have been overlaid. Finally, the data class allows to store a sample point and a Z_stack for the DCEL-component it is attached to. See Section 3.3 for details on the Z_stack. Subsequent operations, as Construct_isolator or Equal_z, can benefit from this data as first, the global computation save repeated local computations within the functors, and second, the best algorithm according to the given data can be triggered directly. Public members of Projection_2 also provide access to the stored information for users. As examples we mention .hassilhouette (Dcel_handle $h$ ) and . has_cut (Dcel_handle h), where Dcel_handle is either a vertex-, edge-, or face-handle. In addition, Projection_2 forwards iterators to traverse all vertices, edges, and faces.

The presented SURFACETRAITS_3 concept in combination with the generic Projection_2 class template enables to write generic and reusable code for different families of surfaces. This comprises in particular the construction of arrangements as theoretically presented in Section 2, i.e., to find proper refinements of involved curves and to attach correct multiplicities, regularities, and degradations to each resulting DCEL-component. To do so, we can identify three subtasks, each of which implemented by a functor.

Construct $\mathcal{A}_{\{S\}}$ : Construct_surface_projection_2 provides all curves of interest for that we can easily construct intermediate arrangements with the help of the provided Arrangement_traits_2 and CGAL's Arrangement_2. Using the extension DCEL-components with P_dcel_data and proper updates of them, we implemented the iterated construction of $\mathcal{A}_{\{S\}}$ as presented in Section 2 in terms of overlays of the intermediate arrangements and subsequent simplification steps. Our implementation is tuned to only perform the necessary steps, e.g., it stops to assign regularities as soon as all DCEL-features know their value.

Construct $\mathcal{A}_{\Sigma_{i, j}}$ : For this task we use the conceptual functor Construct_surface_pair_projection_2 to first compute an intermediate arrangement consisting of the projected cut-curve only. In a second step, we refine it with respect to $\mathcal{A}_{\left\{S_{i}\right\}}$ and $\mathcal{A}_{\left\{S_{i}\right\}}$ using overlays and subsequent simplifications, i.e., removing features not belonging to $\Sigma_{i, j}$. During these overlays we obtain information about the regularity with respect to $\left\{S_{i}\right\}$ and $\left\{S_{j}\right\}$ of DCEL-components containing $\Sigma_{i, j}$. These are required by the final refinement step, namely to refine the arrangement further with respect to curves that influence the degradation values along $\Sigma_{i, j}$.

Overlay of such arrangements: This task is simple if we are only interested in $\mathcal{A}_{\left\{S_{i}, S_{j}\right\}}$, as we only have to omit the simplification step in the previous process during the refinement with respect to $\mathcal{A}_{\left\{S_{i}\right\}}$ and $\mathcal{A}_{\left\{S_{j}\right\}}$. Else, $N>2$ and we have to overlay all $\mathcal{A}_{\left\{S_{i}\right\}}, 1 \leq i \stackrel{\leq}{\leq} s$ and all $\mathcal{A}_{\Sigma_{i, j}} 1 \leq i<j \leq N$. Let us call them basic arrangements. The overlay of curves is directly supported by CGAL's package, which is called inside Overlay_projection_2. Its special focus is to take care about the attached data. It is advantageous that different basic arrange- ment carry independent data, either for a single surface or for a unique pair of surfaces. This observation keeps the merge simple, i.e., we only have to merge the lists of originating key-value-pairs, always distinguishing between single surfaces and pairs of them.

Each functor applies an extensive caching strategies to avoid repeated constructions. This means that for a given surface $S_{i}$, there will be exactly one Projected_2 instance that represents $\mathcal{A}_{\left\{S_{i}\right\}}$, and for $\overline{\mathcal{S}} \subset \mathcal{S}$ there will be exactly one Projected_2 instance that represents $\mathcal{A}_{\overline{\mathcal{S}}}$.

\subsection{Z_stack}

We next explain the other important data structure used to represent what happens in the third dimension. From Section 2 it follows that it suffices to compute the $z$-pattern for a sample point of each DCEL-component of $\mathcal{A}_{\mathcal{S}}$ for given $\mathcal{S}$ or a subset of it, depending on the desired application. For a vertex, there is no choice for the sample point. For an edge, we have a one-dimensional choice. For a face, the choice is even two-dimensional. For edges and faces, it is useful to construct points with rational coordinates of low bit-size, if possible. The implementation already internally provides methods to construct such sample points. We admit, that the complexity of computed sample points can still be improved in a future version, a task that also depends on the used geometry traits class.

Definition 3.1. A Z_stack represents a z-pattern $G=g_{1}, \ldots, g_{k}$ where each $g_{i}$ is augmented by (at least) one isolating interval to approximate the meant induced $z$-coordinate.

In $\mathrm{C}++$, we represent a Z_stack by two entities. First, a list of instances of Z_at_xy_isolator, i.e., one for each involved surface and second, a sorted container of surface-sets, so-called Z_cells. Cells for $\pm \infty$ are not explicitly maintained. A Z_cell stores instances of std: :pair< Surface_3, int $>$, that we call a surface-sheet. Observe that this combination, decouples a $z$ pattern from the geometric information (i.e., $Z_{p, i}$ ), but also allows to connect them. A Z_cell might store sheets for more than one surface, but all have in common that their corresponding intervals of $z$-coordinates are isolating with respect to the intervals of the neighboring cells.

Our algorithm to compute a Z_stack is basically a merge-sort using Algorithm 3.2 to obtain the order of two cells. The mergephase is enhanced with filters that are fed by data stored along with the planar DCEL-components.

Algorithm 3.2. Order of two $Z_{-} c e l l s ~ z c_{1}, z c_{2}$

1. Pick a sheet $<S_{1, i}, k_{1, i}>$ of $z c_{1}$ and $<S_{2, i}, k_{2, i}>$ of $z c_{2}$.

2. If underlying DCEL-component is a face, proceed with (9).

3. If $\Sigma_{1,2}$ is not involved in DCEL-component, proceed with (9).

4. Refine intervals of two the involved isolators for $S_{1}$ and $S_{2}$ until each interval overlaps with at most one interval of the other isolator. The overlapping intervals form a positive candidate list for possible intersections of $S_{1}$ and $S_{2}$ along the current $\ell_{p}$. If no candidate is found, proceed with (9).

5. If DCEL-component is an edge that involves $\Sigma_{1,2}$ and whose multiplicity (degradation) is 1, check whether the single possible overlap matches the cells (i.e., sheets) currently under consideration. If so, return EQUAL, if not, proceed with (9).

6. If DCEL-component is a vertex, select incident edges of $\mathcal{A}_{\left\{S_{1}, S_{2}\right\}}$ whose Z_stack indicate an intersection of $S_{1}$ and $S_{2}$. Compute for each Z_cell containing an intersection the adjacencies towards given vertex, i.e., a pair of Z_cells. If 
the pair of Z_cel Is currently in focus is contained in this list, return EQUAL, which follows by Condition 2.11. Otherwise proceed with (9).

7. If DCEL-component is a vertex, check whether both $\Sigma_{1}$ and $\Sigma_{2}$ are involved. If not, proceed with (9), as only isolated points remain for possible intersections, but intersecting isolated points are indicated by the existence of both projected silhouette-curves.

8. Finally, call Equal_z for $z c_{1}$ and $z c_{2}$. If it returns true, return EQUAL.

9. Reaching here indicates that the cells are detected not to be equal, i.e., they can be refined until they do not overlap any more, which gives the correct order, i.e., SMALLER or GREATER.

We finally want to remark, that the lifting follows the lazy evaluation scheme. This means that sample points for DCELcomponents and their $z$-stacks are only computed on demand. Further requests for them are served by cached versions. Of course, Projection_2 offers public members to access sample points and $z$-stacks of DCEL-components.

When merging attached data due to an overlay, our code always reuses already computed sample points. The same holds for $\mathrm{z}$ stacks and isolators attached to them.

\subsection{Applications}

The complexity of $\mathcal{A}_{\mathcal{S}}$ with all its $z$-stacks is quite high due to the projection and the lifting; even for relatively small $N$. However, the framework can also serve as a key ingredient for concrete applications, e.g., like to compute a surface mesh. To do so, compute a partial vertical decomposition (see [Shaul and Halperin 2002]) of $\mathcal{A}_{\{S\}}$, create its $z$-stacks along with all adjacency information and lift the resulting faces according to their $z$-stacks into the third dimension. The situation on their boundaries is specified by proper $z$-stacks and the computed adjacencies. To obtain a full triangulation, it might be required to split faces further, i.e., with respect to existing crest-lines within a face. The meshing is currently not implemented, in contrast to the following two applications.

\section{Space curves}

Definition 3.3. A space curve is the intersection set of two surfaces $S_{1}, S_{2}$, if at most 1-dimensional.

To represent a space curve, one usually decomposes it into 0and 1-dimensional parts, where 0-dimensional parts form isolated points, while the 1-dimensional arcs can have properties, like $x$ - or $x y$-monotonicity. Our implementation provides $\mathrm{C}++$ class templates called Surface_point_3 and Surface_arc_3. The representation of a point is a tuple (Point_2, Surface_3, int), i.e., a planar base point, a supporting surface, and its sheet-number. $x$ - and $y$-coordinate are given explicitly by the planar point, the $z$-coordinate is encoded implicitly by the other two types. A bounded 1-dimensional arc in 3D is represented by a tuple (Surface_point_3, Surface_point_3, X_monotone_curve_2, Surface_3, int, int, int), where the points encode the ordered lexicographic smallest and largest point of the arc, and the remaining entries lift the planar curve onto the given surface. The ints encode sheet-numbers at the lexicographic smallest and largest point, and in the interior of the arc, where the number must be constant. Note that all three can even be equal or different. Unbounded and vertical arcs are implemented, but omitted in this description.

The following algorithm computes the decomposition of the space-curve defined by $S_{1}, S_{2}$ into isolated vertices and arcs.

\section{Algorithm 3.4. Arcs and points of a space curve}

1. Compute $\mathcal{A}_{\left\{S_{1}, S_{2}\right\}}$ and extract vertices and edges belonging to $\Sigma_{1,2}$.

2. Obtain for each such vertex and each such edge its Z_stack.

3. Compute for each such edge the adjacencies towards its source and target vertex (determines how lifted arcs are connected with lifted vertices).

4. For an edge, the Z_stack and the adjacencies give all information required to construct instances of type Surface_arc_3.

5. Isolated vertices in $3 D$ are detected and constructed by checking whether there exist Z_cells over DCEL-vertices, that are not adjacent to a $\mathrm{Z}_{\mathrm{C}} \mathrm{Cel} l$ of an edge. It remains to construct proper instances of type Surface_point_3 via the available information.

Note that vertical arcs are omitted here, as their detection and representation is much simpler.

A careful reader might detect that this approach requires to compute both $\mathcal{A}_{\left\{S_{1}\right\}}$ and $\mathcal{A}_{\left\{S_{2}\right\}}$. Observe, that the output is not demanding for both surfaces at the same time. It suffices to express the decomposition of a space curve into points and arcs only in terms of the surface with lower complexity, e.g., the algebraic degree of a surface. Let $S_{1}$ be the surface with lower complexity.

We next show how to avoid the computation of $\mathcal{A}_{\left\{S_{2}\right\}}$ and $\mathcal{A}_{\left\{S_{1}, S_{2}\right\}}$, but at the cost of an additional functor in the SURFACETRAITS_3 concept, called Common_z. In contrast to Equal_z, which only checks the equality for given intervals, Common_z constructs a new instance of type Z_at_xy_isolator that represents the common interesting $z$-coordinates of Surface_3 surface 1 and Surface_ 3 surface 2 along a vertical line defined by the given Point_2 point. Due to lacking $\mathcal{A}_{\left\{S_{2}\right\}}$ and $\mathcal{A}_{\left\{S_{1}, S_{2}\right\}}$, we do not have access to full knowledge about multiplicities, regularities, and degradations with respect to $\left\{S_{2}\right\}$ and $\left\{S_{1}, S_{2}\right\}$. Thus, Common_z has to deal without these information. It depends on the family of surfaces, how to compute it. We also need a new algorithm to construct the Z_stack.

Algorithm 3.5. Z_stack for a DCEL-component being part of $\Sigma_{1,2}$ within overlay of $\mathcal{A}_{\left\{S_{1}\right\}}$ and $\mathcal{A}_{\Sigma_{1,2}}$

1. Construct Z_at_xy_isolator isolator 1 for $S_{1}$ using Construct_isolator.

2. Construct Z_at_xy_isolator isolator12 for intersections of $S_{1}$ and $S_{2}$ using Common_z.

3. Refine intervals of isolator 12 until each is included in an interval of isolator 1 .

4. Create Z_cell for each interval of isolator 1 and add $S_{2}$ to cell, if interval overlaps with an interval of i solator 12 . Observe that no sheet-number for $S_{2}$ is available (but also not needed).

We have implemented this output-sensitive strategy in a classtemplate called SoX: : Curve_3. We consider this as a basic implementation that can be used whenever space curves are computed using its projection onto the $x y$-plane. In this light, this work can be seen as a prototypical implementation of a key ingredient for an upcoming Curved_kernel_3 in CGAL. Section 4 reports on experiments where this approach decomposes intersection curves of quadrics to compute arrangements on quadrics. 
Lower envelopes Regard the surfaces in $\mathcal{S}$ as functions in $x$ and $y$ that return for given $p=\left(p_{x}, p_{y}\right)$ the smallest $z$-coordinate of the surface's intersections with $\ell_{p}$.

Definition 3.6 (Lower Envelope). The lower envelope $\mathcal{E}_{\mathcal{S}}$ of $\mathcal{S}$ is the point-wise minimum of these functions: $\mathcal{E}_{\mathcal{S}}(x, y):=$ $\min S_{i}(x, y)$, where the minimum is taken over all functions defined at $(x, y)$.

The minimization diagram $\mathcal{M}_{\mathcal{S}}$ is one way to represent $\mathcal{E}_{\mathcal{S}}$ as the subdivision of $\mathbb{R}^{2}$ into maximal connected cells such that $\mathcal{E}_{\mathcal{S}}$ is attained by a fixed (possibly empty) subset of functions over the interior of each cell. Meyerovitch presented a generic implementation of a divide-and-conquer algorithm based on CGAL's Arrangement_2 package [Meyerovitch 2006]. The algorithm requires to be instantiated with an extension of CGAL's ARRANGEMENTTRAITS_2 concept. This ENVELOPETRAITS_3 concept additionally demands 3D-types and operations on them. With our framework we provide a generic implementation of such a model, that we are calling SoX: : Surface_3_envelope_traits.

As types, Surface_3 and Xy_monotone_surface_3 are expected. We map both to the Surface_3 type defined in the used model of the SURFACETRAITS_3 concept. This may be surprising at first, since a surface in general is not $x y$-monotone. However, it is only an implementation detail to simplify matters. All later operations that work on an $x y$-monotone surface $S_{i}$ consider only the lowest part of the surface. In this light, the required implementation of Make_xy_monotone_3 is simple. Two construction functors implement the required projections.

Construct_projected_boundary_2: Computes for a given $S_{i}$ of type Xy_monotone_surface_3 its projected boundary. To provide this information, we compute $\mathcal{A}_{\left\{S_{i}\right\}}$ and traverse its edges vertices. We discard an edge, if its Z_stack is empty. If not, compute the Z_stacks of its two incident faces. If one stack is empty and the other not, the edge is considered as a projected boundary and returned. The non-empty stack determines on which side of the edge, the surface exists. Vertices are reported if their Z_stack contains $S_{i}$ in their lowest cells, and this cell is not adjacent to a cell of the incident faces' Z_stacks.

Construct_projected_intersection_2:

computes the projected intersection curves of two Xy_monotone_surface_3s, called $S_{i}$ and $S_{j}$. We compute the overlay of $\mathcal{A}_{\left\{S_{i}, S_{j}\right\}}$. Next, we traverse all edges (and isolated vertices) discarding those not participating in $\Sigma_{i, j}$, those with an empty Z_stack, and those whose lowest Z_cell does not contain $S_{i}$ and $S_{j}$. The remaining edges and vertices are returned.

The concept also requires to implement functors that compare the relative alignment of two Xy_monotone_surface_3s in $z$ direction above a point, above a curve, or above a face incident to a projected intersection curve, that is a sub-face of the projected curve boundaries. Obviously, such a vertical alignment is encoded in a Z_stack of the appropriate $\mathcal{A}_{\left\{S_{i}, S_{j}\right\}}$. Our task is to find the correct one which itself reduces to perform a point location for a given point, or for a constructed point on a curve.

To summarize: Using the this generic model of the ENVELOPETRAITS_3 concept, computing (lower) envelopes for a family of surfaces boils down to provide a model of the SURFACETRAITS_3 concept for that class of surfaces. We admit that a specialized model for lower envelopes might be more efficient, but obviously lacks of the possibility to support other (introduced) applications. Section 4 discusses our model of the SURFACETRAITS_3 concept for quadrics along with initial experiments.

\section{The model for quadrics}

We implemented in EXACUS' QUADRIX library the model for quadrics that fulfills the SURFACETRAITS 3 concept.
Definition 4.1. A quadric is an algebraic surface of degree 2. It is given as the vanishing set of a trivariate polynomial $f(x, y)=$ $a_{2}(x, y) z^{2}+a_{1}(x, y) z+a_{0}(x, y)$ in $\mathbb{R}^{3}$.

We typedef Surface_3 to QUADRIX's existing class template QdX: : Quadric_3. Elimination theory gives the following results.

Corollary 4.2. Let $f_{1}, f_{2}$ be square-free and coprime polynomials defining quadrics $Q_{i}=V\left(f_{i}\right), i=1,2$. The projected silhouettecurve $\Sigma_{i}$ of $Q_{i}$ is given by $\operatorname{res}_{z}\left(f_{i}, f_{i z}\right)=\operatorname{sr}_{0}\left(f_{i}, f_{i z}, z\right)$ which defines a real plane algebraic curve of degree at most 2 . The projected intersection curve of $Q_{1}$ and $Q_{2}$, is given by $\operatorname{res}_{z}\left(f_{1}, f_{2}\right)=$ $\operatorname{sr}_{0}\left(f_{1}, f_{2}, z\right)$ which is a real plane algebraic curve of degree at most 4 .

Our model typedefs Arrangement_traits_2 to a generic model of CGAL's ARRANGEMENTTRAITS 2 concept defined in EXACUS's SWEEPX library. It is parameterized in the curveanalysis and the analysis of pairs of curves. Instead of the specialized classes for quadrics [Berberich et al. 2005b], we instantiate it with an implementation for algebraic curves of arbitrary degree. The corresponding analysis classes are implemented in EXACUS's ALCIX library and details about it can be found in recent publications [Eigenwillig et al. 2007; Eigenwillig and Kerber 2008]. Their main source of efficiency consists in a careful combination of symbolic and approximate computations, without sacrificing the correctness of the overall result. The important reason for this switch is the possibility to approximate a point's $x$ - and $y$-coordinate as intervals with arbitrary precision. This is crucial, as we want to use the Bitstream Descartes method [Eigenwillig et al. 2005] as our Z_at_xy_isolator type. This methods isolates the real roots of a polynomial $g(t)$ that is square-free and whose coefficients are "bit-streams", i.e., arbitrary real numbers that are refineable to any positive absolute error. The method itself controls automatically the necessary approximation precision. This exactly serves our goal, as we want to isolate the real roots of $f\left(p_{x}, p_{y}, z\right) \in \mathbb{R}[z]$ with $p=\left(p_{x}, p_{y}\right) \notin V L_{i}$.

The functor Construct_surface_curves_2 has to provide three operators for a given surface ( $\left.\mathrm{Sf}_{-} 3\right)$. For simplicity we abuse notation and unify surface and its defining polynomial. We also define for $f=\sum_{i=0}^{n} a_{i} z^{i}$ and $m<n: f^{(m)}:=\sum_{i=0}^{m} a_{i} z^{i}$. The implementation of the functor uses operations on polynomials from EXACUS' NUMERIX library to obtain the desired polynomials (see list) and to compute their (cached) square-free factorization. For each factor we construct a cached std: : pair consisting of an instance of AcX: : Algebraic_curve_2 and an int representing its corresponding multiplicity. Each such pair is returned through an Output Iterator (OI).

OI operator() ( $\mathrm{Sf}_{-} 3 \mathrm{f}$, OI Oi)

compute and use $\operatorname{res}_{z}\left(f, f_{z}\right)$

OI operator() ( $\mathrm{Sf}_{-} 3 \mathrm{f}$, int $\mathrm{m}$, OI oi)

obtain and use $a_{m}$ with $0 \leq m \leq 2$.

OI operator() (Sf_3 $\mathrm{f}$, int $\mathrm{m}$, int $i$, OI Oi)

compute and use $s r_{i}\left(f^{(m)}, f_{z}^{(m)}, z\right)$ with $0 \leq i<m \leq 2$.

For the Construct_surface_pair_curves_2 functor, exactly the same approach is taken, with the difference that the desired polynomials are expressed with respect to two given surfaces.

OI operator() (Sf_3 f1, Sf_3 f2, OI oi)

compute and use $\operatorname{res}_{z}\left(f_{1}, f_{2}\right)$

OI operator() (Sf_-3 f1, int $\mathrm{m} 1, \mathrm{Sf}_{-} 3 \mathrm{f} 2$, int $\mathrm{m} 2$, int $i$, OI oi) 


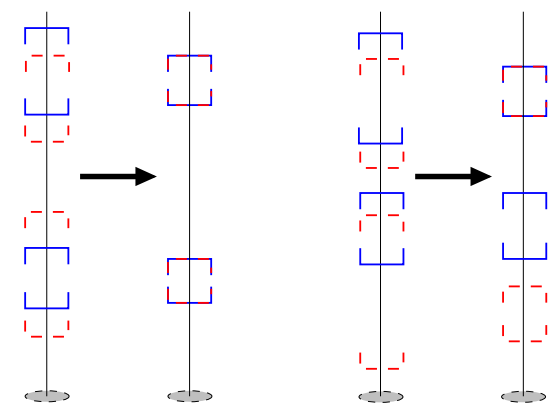

Figure 1: Equal_z: Illustration of cases 2 (left) and 3 (right), roots of Isolator 1 are blue, roots of Isolator 2 are red.

compute and use $s r_{i}\left(f_{1}^{\left(m_{1}\right)}, f_{2}^{\left(m_{2}\right)}, z\right), 0 \leq i<\min \left(m_{1}, m_{2}\right) \leq$ 2

These two functors actually are not specific to quadratic algebraic surfaces. They can be used for algebraic surfaces of arbitrary degree, in contrast to the implementations of the other three required functors. We first assume that no vertical line is contained in a quadric.

Lemma 4.3. The polynomial $f\left(p_{x}, p_{y}, z\right) \in \mathbb{R}[z]$ has either no real root, a double real root, or two distinct real roots. If it has a double root, then $\operatorname{res}_{z}\left(f, f_{z}\right)\left(p_{x}, p_{y}\right)=0$. Contrary if $\operatorname{res}_{z}\left(f, f_{z}\right)\left(p_{x}, p_{y}\right)=0$ then the $z$-stack at $p$ contains at most one finite point.

Proof. The first assertion is rather trivial. If $z_{0}$ is a multiple root of $f\left(p_{x}, p_{y}, z\right)$ then it is also a root of $f_{z}\left(p_{x}, p_{y}, z\right)$, thus $\operatorname{res}_{z}\left(f, f_{z}\right)\left(p_{x}, p_{y}\right)=0$. For $a_{2}(x, y)=0$ and $\ell_{p} \not \subset Q$ the backward direction is trivial as in this case $f(x, y, z)$ is a polynomial of degree one or less in $z$ for all $(x, y)$. If $a_{2}(x, y) \neq 0$ and $\operatorname{res}_{z}\left(f, f_{z}\right)\left(p_{x}, p_{y}\right)=0$ the polynomials $f\left(p_{x}, p_{y}, z\right)$ and $f_{z}\left(p_{x}, p_{y}, z\right)$ must share a common root $z_{0}$, thus for $\ell_{p} \not \subset Q$, the $z$-stack $Z_{p}$ is given by $\left\{z_{0}\right\} \cup\{ \pm \infty\}$. In case where $Q$ contains the vertical line at $p$, we refer to paragraph about vertical lines.

Construct_isolator: Using Lemma 4.3 we isolate with the Bitstream Descartes method the real roots of $f\left(p_{x}, p_{y}, z\right)$ in case $p$ 's degradation is 0 or of $f_{z}\left(p_{x}, p_{y}, z\right)$ if $p$ 's degradation is greater than 0 . Observe that both polynomials fulfill the demanded property of being square-free.

Equal_z: Given two surface-sheets and isolators (i.e., surface1/2, isolator1/2, and int1/2). Task: Decide whether the indicated $z$-approximations are equal. From Algorithm 3.2 we can assume the following when being called. Each interval overlaps with at most one interval or the other isolator and at least one of these overlaps states a true equality. Three cases are left for the two involved quadrics.

1. If some $\Sigma_{i}$ exists, the single overlap is the only possible and demanded. Thus return true.

2. Both $f_{1}(x, y, z)=a_{2}(x, y) z^{2}+a_{1}(x, y) z+a_{0}(x, y)$ and $f_{2}(x, y, z)=b_{2}(x, y) z^{2}+b_{1}(x, y) z+b_{0}(x, y)$ have two distinct real roots and they are equal at the given $p$, i.e., there exists a constant $c \in \mathbb{R} \backslash\{0\}$ with $f_{1}\left(p_{x}, p_{y}, z\right)=$ $c f_{2}\left(p_{x}, p_{y}, z\right)$. This is exactly the case if the two vectors

$$
\left(a_{2}\left(p_{x}, p_{y}\right), a_{1}\left(p_{x}, p_{y}\right), a_{0}\left(p_{x}, p_{y}\right)\right)^{T}
$$

and

$$
\left(b_{2}\left(p_{x}, p_{y}\right), b_{1}\left(p_{x}, p_{y}\right), b_{0}\left(p_{x}, p_{y}\right)\right)^{T}
$$

are linear equivalent, which can be checked by $\left(a_{0} b_{1}-\right.$ $\left.a_{1} b_{0}\right)\left(p_{x}, p_{y}\right)=0 \wedge\left(a_{0} b_{2}-a_{2} b_{0}\right)\left(p_{x}, p_{y}\right)=0 \wedge\left(a_{1} b_{2}-\right.$ $\left.a_{1} b_{2}\right)\left(p_{x}, p_{y}\right)=0$. The checks are implemented as considering $h_{i j}:=a_{i} b_{j}-a_{j} b_{i}$ as planar curves, and test whether $p$ lies on them. This is a basic tool provided by EXACUS' ALCIX library, which is even filtered by interval arithmetic. If all conditions hold, i.e., two common roots exists (out of two possible), return true.

3. Otherwise, two candidate pairs remain for a single equality. We refine them in parallel, until only one candidate is left. If the given values for root 1 and root 2 correspond to that candidate, return true, else return false.

Adjacency: This functor is implemented mostly combinatorially. If given incident DCEL-components consist of an edge and a vertex, the only possible adjacency is $\langle 0,0\rangle$ as the silhouettecurve of a quadric lies in the plane defined by $f_{z}$. Assume now that isolator 1 belongs to a face. If $r_{1}=0$, no adjacency can be reported. If $r_{1}=2$, all incident components belong to the $\Sigma$. For both $k=0,1$ we return $\langle 0,0\rangle$. If $r_{1}=1$, the fact that the face's boundary exists allows to conclude by Lemma 4.3 that $r_{2}<0$. This either implies to return $\langle 0,-1\rangle$ or $\langle 0,1\rangle$ to indicate an infinite behavior, or to return $\langle 0,0\rangle$, if the quadric is vertical at the sample point of isolator 2 . To distinguish the infinity cases, it is required to obtain the sign of the root of $f_{z}\left(p_{x}, p_{y}, z\right) \in \mathbb{R}[z]$, for the sample point $p$ of isolator 1 . Last, if isolator 2 belongs to a face, Lemma 4.3 gives that $r_{1} \leq 1$. If $r_{1}=0$, no adjacency exist. Else, three cases are left: (1) $\bar{r}_{2}=0$, the output consists of no pair (2) $r_{2}=1$, return the pair $\langle 0,0\rangle$. (3) $r_{2}=2$, the list contains two pairs, namely $\langle 0,1\rangle$, and $\langle 1,1\rangle$

Vertical lines A quadric $Q$ contains a vertical line $\ell_{p}$ at $p \in \mathbb{R}^{2}$ exactly if $a_{2}(x, y)=0$ and $p$ is an intersection point of the line $L=V\left(a_{1}(x, y)\right)$ and the conic $C=V\left(a_{0}(x, y)\right)$. Then for each point $(x, y) \notin L$, there exists a unique lift $(x, y, z) \in Q$ with $z=-\frac{a_{0}(x, y)}{a_{1}(x, y)}$. Furthermore, there exists no point on $Q$ above any $(x, y) \in L \backslash(L \cap C)$ and for each (of at most 2) intersection point $p \in L \cap C$ the quadric contains the vertical line $\ell_{p}$. The arrangement $\mathcal{A}_{\{Q\}}$ as defined in Section 2 is quite simple in this situation: The projected silhouette-curve $L$ divides $\mathbb{R}^{2}$ into two half-planes, which are the faces $F_{1}$ and $F_{2}$ of $\mathcal{A}_{\{Q\}}$. The intersection points $L \cap$ $C$ represents all vertices in $\mathcal{A}_{\{Q\}}$ and they decompose $L$ into (at most 3 ) edges. As the edges cannot be lifted onto $Q$, no adjacency relationship between them and vertices can be reported.

In the following steps we will show how to determine the stack $Z_{p}=\left\{-\infty=z_{p,-1}, z_{p, 0}, \ldots, z_{p, m_{p}}, z_{p, m_{p}}=+\infty\right\}$ and how to get the adjacency information as described in Section 2, condition (4). In Theorem 2.21 we have already proven that for each face $F=F_{1}$ or $F=F_{2}$ there exists a corresponding interval $I_{F}$ such that for each $z^{*} \in I_{v}$ we have a sequence $p_{n} \in F$, converging against $p$, with $z^{*}=\lim _{n \rightarrow \infty} z_{p_{n}}=\lim _{n \rightarrow \infty}-\frac{a_{0}\left(p_{n}\right)}{a_{1}\left(p_{n}\right)}$.

From an affine change of coordinates we can assume that $L=$ $V(y)$, i.e., $L$ is the $x$-axis. Writing $a_{0}(x, y)=c_{0} x^{2}+c_{1} y^{2}+$ $c_{2} x y+c_{3} x+c_{4} y+c_{5}$ with variable coefficients $c_{i} \in \mathbb{R}$, for the $z$-value of any $(x, y, z) \in Q$ we have

$$
z=\frac{c_{0} x^{2}+c_{1} y^{2}+c_{2} x y+c_{3} x+c_{4} y+c_{5}}{y}
$$

For a fixed $y \neq 0$ the set of $z$-values is given by a parabola $\left(c_{0} \neq 0\right)$, which has its unique local extremum $z_{\max , y}$ at the point $x_{\max }$ with

$$
2 c_{0} x_{\max , y}+c_{2} y+c_{3}=0
$$

Thus we get $x_{\max , y}=\frac{-c_{3}-c_{2} y}{2 c_{0}}$ and

$$
z_{\max , y}=\frac{4 c_{0} c_{5}+4 y c_{0} c_{4}-2 y c_{2} c_{3}-c_{3}^{2}+4 y^{2} c_{0} c_{1}-y^{2} c_{2}^{2}}{4 y c_{0}}
$$


Now we distinguish three cases:

1. $c_{0}=0: C$ and $L$ intersect in a unique point $p=\left(-\frac{c_{5}}{c_{3}}, 0\right)$. Now given an arbitrary $z^{*} \in \mathbb{R}$, we have

$$
f\left(x_{y}, y, z^{*}\right)=0 \Leftrightarrow x_{y}\left(c_{2} y+c_{3}\right)=y z^{*}-c_{1} y^{2}-c 4 y-c 5
$$

For $y \rightarrow 0$ we get $x_{y} \rightarrow-\frac{c_{5}}{c_{3}}$, thus the conic $C_{z^{*}}$, which is implicitly given by the equation $x\left(c_{2} y+c_{3}\right)=y z^{*}-c_{1} y^{2}-$ $c 4 y-c 5$, passes the point $p$ and the face $F$. It follows the existence of a sequence $p_{n} \rightarrow p \in V\left(a_{0}\right) \cap V\left(a_{1}\right)$ with $z_{p_{n}} \rightarrow z^{*}$.

2. $\left|V\left(a_{0}\right) \cap V\left(a_{1}\right)\right|=0$ or 2 and $c_{0} \neq 0$ : For a fixed $z^{*} \in \mathbb{R}$, the conic $C_{z^{*}}=V\left(f\left(x, y, z^{*}\right)\right)$ has exactly two intersection points with $L$. Hence, $C_{z^{*}}$ has only ordinary intersections with $L$. Thus, $C_{z^{*}}$ contains an arc that passes $V\left(a_{0}\right) \cap V\left(a_{1}\right)$ and the face $F$. It follows the existence of a sequence $p_{n} \rightarrow$ $p \in V\left(a_{0}\right) \cap V\left(a_{1}\right)$ with $z_{p_{n}} \rightarrow z^{*}$.

3. $\left|V\left(a_{0}\right) \cap V\left(a_{1}\right)\right|=1$ : In this case the quadratic polynomial $a_{0}(x, 0)$ has a multiple root and $p=V\left(a_{0}\right) \cap V\left(a_{1}\right)=$ $\left(\frac{-c_{3}}{2 c_{0}}, 0\right)$. Hence, we get $c_{3}^{2}-4 c_{5} c_{0}=0$ and furthermore

$$
\begin{gathered}
\lim _{y \rightarrow 0} z_{\max , y}=\lim _{y \rightarrow 0} \frac{4 y c_{0} c_{4}-2 y c_{2} c_{3}+4 y^{2} c_{0} c_{1}-y^{2} c_{2}^{2}}{4 y c_{0}} \\
=\frac{2 c_{0} c_{4}-c_{2} c_{3}}{2 c_{0}}
\end{gathered}
$$

Now, the line $L_{\max }$, implicitly given by $2 c_{0} x+c_{2} y+c_{3}=0$ passes the point $p$ and contains a sequence of points $p_{n} \rightarrow p$ with $z_{p_{n}} \rightarrow \frac{2 c_{0} c_{4}-c_{2} c_{3}}{2 c_{0}}=: z_{p, 0}$ for $n \rightarrow \infty$. In the next step we will show that for any other sequence $p_{n}^{\prime} \rightarrow p$ we must get $z_{p_{n}} \rightarrow z^{\prime} \geq z_{p, 0}$ or $\leq z_{p, 0}$ depending on whether $c_{0}>0$ or $c_{0}<0$. W.1.o.g. we assume that $c_{0}>0$. Then for a fixed $y$ the parabola $\frac{c_{0} x^{2}+c_{1} y^{2}+c_{2} x y+c_{3} x+c_{4} y+c_{5}}{y}$ has a global minimum $z_{\max , y}$ at $x_{\max , y}$. Thus, for any point $p_{n}^{\prime}=\left(x_{n}^{\prime}, y_{n}^{\prime}\right)$ we must have $z_{p_{n}^{\prime}}=-\frac{a_{0}\left(x_{n}^{\prime}, y_{n}^{\prime}\right)}{a_{1}\left(x_{n}^{\prime}, y_{n}^{\prime}\right)} \geq z_{\max , y_{n}^{\prime}}$. It follows that $\lim _{p_{n}^{\prime} \rightarrow p} z_{p_{n}^{\prime}} \geq \lim _{p_{n}^{\prime} \rightarrow p} z_{\max , y_{n}^{\prime}}=z_{p, 0}$.

In the two cases (1) and (2) we have shown that the lifts of the two faces $F_{1}$ and $F_{2}$ are both adjacent to any point on the vertical line $\ell_{p}$, i.e., for any $\left(p, z^{*}\right) \in \ell_{p}$ there exist sequences $p_{n}^{(j)} \in F_{j}$ with $\left(p_{n}^{(j)}, z_{p_{n}^{(j)}}\right) \rightarrow\left(p, z^{*}\right)$ for $j=1,2$. Thus, it suffices that $Z_{p}=\{ \pm \infty\}$, i.e., Construct_isolator returns an empty instance and so Equal_z is trivial. It is clear that Adjacency returns for an incident face $F$ towards $p$ the pairs $<0,-1>$ and $<0,0>$.

In the third case, for exactly all $z^{*}$ in between $\frac{2 c_{0} c_{4}-c_{2} c_{3}}{2 c_{0}}$ and $\pm \infty$ (depending on whether $c_{0}>0$ or $c_{0}<0$, respectively) there exists a sequence $p_{n}^{(j)} \in F_{j}$ with $p_{n}^{(j)} \rightarrow p$ and $\left.z_{p_{n}^{(j)}}\right) \rightarrow z^{*}$. As we can also pursue the affine coordinate transformation in case where $f$ is given with arbitrary variable coefficients, it is possible to get formulas in terms of these coefficients to decide in which case we are and to determine the single non-infinity entry $z_{p, 0}$ of $Z_{p}$. Observe that $p=\left(p_{x}, p_{y}\right)$ and $z_{p, 0}$ are all rational. Thus, Equal_z can be implemented in terms of rational arithmetic. Adjacency returns for an incident face towards the vertical line at $p$, either the pairs $\langle 0,-1\rangle,\langle 0,0\rangle$ or the pairs $\langle 0,0\rangle,\langle 0,1\rangle$, depending on the sign of $c_{0}$ and the face.

\begin{tabular}{r|rr|rr}
$\sharp$ Surfaces & $\sharp$ DCEL & $\sharp \mathrm{z}$-cells & $\mathrm{t}$ & $\mathrm{t} /$ cell \\
\hline 2 ellipsoids & 13 & 12 & $\mathbf{0 . 1 s}$ & $5.7 \mathrm{~ms}$ \\
4 ellipsoids & 230 & 904 & $\mathbf{2 . 8 s}$ & $3.4 \mathrm{~ms}$ \\
6 ellipsoids & 877 & 5942 & $\mathbf{1 9 . 9 s}$ & $3.7 \mathrm{~ms}$ \\
8 ellipsoids & 2780 & 25220 & $\mathbf{1 7 1 . 9 s}$ & $7.2 \mathrm{~ms}$ \\
10 ellipsoids & 4952 & 52788 & $\mathbf{5 8 2 . 0 s}$ & $11.5 \mathrm{~ms}$ \\
\hline 2 quadrics & 53 & 160 & $\mathbf{0 . 4}$ & $2.7 \mathrm{~ms}$ \\
4 quadrics & 1099 & 7172 & $\mathbf{1 9 . 7}$ & $3.0 \mathrm{~ms}$ \\
6 quadrics & 3946 & 39254 & $\mathbf{1 9 4 . 4}$ & $5.4 \mathrm{~ms}$ \\
8 quadrics & 9983 & 132352 & $\mathbf{2 3 0 6 . 1}$ & $18.1 \mathrm{~ms}$
\end{tabular}

Table 1: Typical performance measures for sets of ellipsoids and arbitrary quadrics of increasing size.

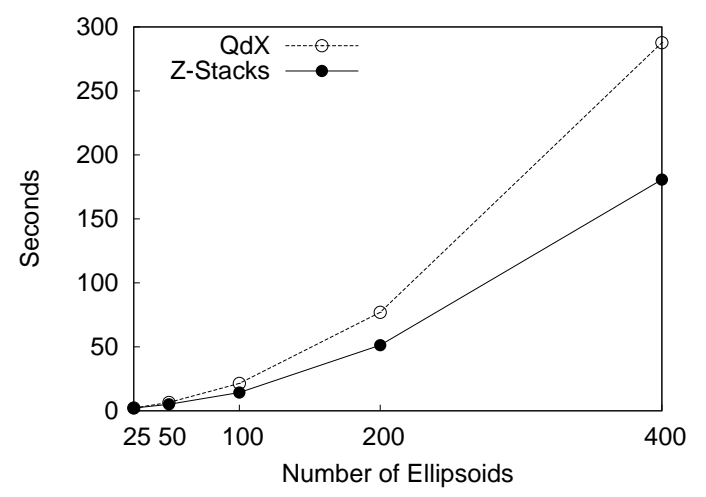

Figure 2: Running times to compute arrangements on an ellipsoid intersected by other ellipsoids.

\section{Results}

We tested the performance of the framework for quadrics by computing all $z$-stacks and all adjacencies for $\mathcal{A}_{\mathcal{S}}$, where $|\mathcal{S}|$ increases. All experiments are executed on a Pentium IV CPU with $3.0 \mathrm{GHz}$ clock-speed and $2 \mathrm{MB}$ of cache. The executables are compiled with gnu's $\mathrm{C}++-$-compiler in version 3.3 with disabled debugging (-DNDEBUG) and enabled optimizations (-O2) and the exact number types of LEDA. Table 1 lists example runs.

It cannot be hidden, that $|\mathcal{S}|$ seems quite small, but on the other hand, the size of the output grows rapidly. For 8 quadrics we already have to compute nearly $10.000 z$-stacks containing more than 130.000 cells, numbers much larger than the ones for two surfaces. On the contrary, the time spent per cell grows much slower. In fact, we have to see an increasing number here, as by construction of the data, similar intervals along each $\ell_{p}$ are intersected by a growing number of quadrics, i.e., it requires additional time to isolate the cells against each other. These first experiments lead to the recommendation to use the framework especially for cases where $|\mathcal{S}|$ is small, like in the next example, where only pairs or triples of surfaces are needed.

We also tested the framework when computing arrangements on a reference quadric induced by intersections with other quadrics. As example we chose increasing sets of random ellipsoids. Figure 2 shows the improvement of about $30 \%$, compared to a former implementation [Berberich et al. 2007]. For general quadrics the ratio is similar. We note that an example arrangement induced by 400 ellipsoids intersecting the reference ellipsoids consists of about 38.000 vertices and 74.000 edges, which is now possible to compute in around 180 s instead of $287 \mathrm{~s}$. These results imply to analyze the performance of the framework with more extensive experiments. 


\section{Conclusion and outlook}

We presented a generic framework, implemented in $\mathrm{C}++$, based on CGAL's Arrangement_2 package to compute $z$-stacks of (algebraic) surfaces and adjacencies between $z$-stacks. The design of it is simple, the interface intuitive and the approach taken does not enforce to assume generic position. A new family of surfaces can be used by implementing a small set of tasks collected in a newly introduced SURFACETRAITS_3 concept. Our first model provides the full functionality for quadrics, i.e., algebraic surfaces of degree 2 being developed within the EXACUS-project [Berberich et al. 2005a]. As the number of $z$-cells grows fast, we see the main application of this tool in providing information for a small set of surfaces, i.e., to compute the topology (and geometry) of a single surface, a single space-curve, or to serve as a key ingredient for high-level algorithms like the computation of envelopes, or threedimensional arrangements. Some of them are already presented and implemented, others are open for future research, in particular, how to reduce the complexity for certain applications. The next goal is to negotiate in detail, how to query the framework instantiated with quadrics to support rotational robot motion planning, which is an intermediate step towards a full support for Piano Mover's instances.

Besides the applications there are also more framework-related open problems. It would be beneficial to simplify the conditionsr. This should enable to model other families of surfaces as well. In addition, it helps to describe the individual steps more generically. We are already working to release the binding to algebraic surfaces and to come up with a much more generic version.

Concerning models, the current challenge to solve is to provide a full model of our SURFACETRAITS_3 concept for algebraic surfaces of arbitrary degree. This model should efficiently implement all the simple operations. A clever combination of cheap approximative and unavoidable costly symbolic computations together with the full set of modular, floating-point-, modular- and geometric filters is promising to be successfull. The case of a single surface is discussed in [Berberich et al. 2008]. If completed, another goal is to implement a model for surfaces composed of patches of algebraic surfaces.

Acknowledgements We thank Michael Kerber for hours of fruitful discussions and for giving comments on a preliminary version of this paper.

\section{References}

Agarwal, P. K., AND Sharir, M. 2000. Arrangements and their applications. In Handbook of Computational Geometry, J.R. Sack and J. Urrutia, Eds. Elsevier, 49-119.

Arnon, D. S., Collins, G. E., ANd McCallum, S. 1984. Cylindrical algebraic decomposition i+ii. SIAM Journal on Computing 13, 865-889. Reprinted in [Caviness and Johnson 1998], pp. 136-165.

Austern, M. H. 1999. Generic Programming and the STL. Addison-Wesley.

Basu, S., Pollack, R., AND Roy, M.-F. 2006. Algorithms in Real Algebraic Geometry, 2nd ed., vol. 10 of Algorithms and Computation in Mathematics. Springer.

Bentley, J. L., And Ottmann, T. A. 1979. Algorithms for reporting and counting geometric intersections. IEEE Transactions on Computers C-28, 643-647.

Berberich, E., Eigenwillig, A., Hemmer, M., Hert, S., Ketther, L., Mehlhorn, K., Reichel, J., Schmitt, S., SCHÖMER, E., AND WOLPERT, N. 2005. Exacus: Efficient and exact algorithms for curves and surfaces. In Proceedings of the 13th Annual European Symposium on Algorithms (ESA 2005), Springer, vol. 3669 of $L N C S, 155-166$.

Berberich, E., Hemmer, M., Kettner, L., Schömer, E., AND WOLPERT, N. 2005. An exact, complete and efficient implementation for computing planar maps of quadric intersection curves. In 21st Annual Symposium on Computational Geometry (SCG'05), ACM, Pisa, Italy, J. Mitchell, G. Rote, and L. Kettner, Eds., Association for Computing Machinery (ACM), 99-106.

Berberich, E., Fogel, E., Halperin, D., Mehlhorn, K., AND WEIN, R. 2007. Sweeping and maintaining twodimensional arrangements on surfaces: A first step. In ESA, Springer, L. Arge, M. Hoffmann, and E. Welzl, Eds., vol. 4698 of Lecture Notes in Computer Science, 645-656.

Berberich, E., Kerber, M., And SAgraloff, M. 2008. Exact geometric-topological analysis of algebraic surfaces. In Proceedings of the 24th Annual Symposium on Computational Geometry (SCG 2008). to appear.

Bredon, G. E. 1993. Topology and Geometry. Springer.

Caviness, B. F., And Johnson, J. R., Eds. 1998. Quantifier Elimination and Cylindrical Algebraic Decomposition, Texts and Monographs in Symbolic Computation. Springer.

Cheng, J.-S., Gao, X.-S., And Li, M. 2005. Determining the topology of real algebraic surfaces. In IMA Conference on the Mathematics of Surfaces, Springer, R. R. Martin, H. E. Bez, and M. A. Sabin, Eds., vol. 3604 of Lecture Notes in Computer Science, 121-146.

Eigenwillig, A., ANd Kerber, M. 2008. Exact and efficient 2d-arrangements of arbitrary algebraic curves. In Proceedings of the Nineteenth Annual ACM-SIAM Symposium on Discrete Algorithms (SODA08), 122-131.

Eigenwillig, A., Kettner, L., Krandick, W., Mehlhorn, K., Schmitt, S., AND Wolpert, N. 2005. A Descartes algorithm for polynomials with bit-stream coefficients. In 8th International Workshop on Computer Algebra in Scientific Computing (CASC 2005), vol. 3718 of LNCS, 138-149.

Eigenwillig, A., Kerber, M., And Wolpert, N. 2007. Fast and exact geometric analysis of real algebraic plane curves. In Proocedings of the 2007 International Symposium on Symbolic and Algebraic Computation (ISSAC 2007), C. W. Brown, Ed., $151-158$.

Gatellier, G., Labrouzy, A., Mourrain, B., And TÉCOURT, J.-P. 2005. Computing the topology of 3dimensional algebraic curves. In Computational Methods for Algebraic Spline Surfaces, Springer, 27-44.

KetTNER, L. 2006. Reference counting in library designoptionally and with union-find optimization. In Proceedings of the First International Workshop on Library-Centric Software Design, LCSD'05, Rensselaer Polytechnic Institute, Computer Science Department, San Diego, CA, USA, D. Musser and J. Siek, Eds., vol. 06-12 of Technical Report, 34-43.

LATOMBE, J.-C. 1993. Robot motion planning, 3rd printing ed., vol. 0124; of Kluwer international series in engineering and computer science; SECS. Kluwer.

Massey, W. 1967. Algebraic Topology: An Introduction. Springer. 
Meyerovitch, M. 2006. Robust, generic and efficient construction of envelopes of surfaces in three-dimensional space. In Proceedings of 14th European Symposium on Algorithms (ESA), vol. 4168 of $L N C S, 792-803$.

MourRain, B., AND TÉCOURT, J.-P. 2005. Isotopic meshing of a real algebraic surface. Technical Report 5508, INRIA SophiaAntipolis.

Mourrain, B., Técourt, J.-P., And Teillaud, M. 2005. On the computation of an arrangement of quadrics in $3 \mathrm{~d}$. Comput. Geom. Theory Appl. 30, 2, 145-164.

Schwartz, J. T., Sharir, M., And Hopcroft, J. E., Eds. 1987. Planning, geometry, and complexity of robot motion. Ablex series in artificial intelligence. Ablex Publ.

ShaUl, H., AND HaLPerin, D. 2002. Improved construction of vertical decompositions of three-dimensional arrangements. In SCG '02: Proceedings of the eighteenth annual symposium on Computational geometry, ACM, New York, NY, USA, 283-292.

Wein, R., Fogel, E., Zukerman, B., and Halperin, D. 2007. 2d arrangements. In CGAL User and Reference Manual, C. E. Board, Ed., 3.3 ed.

Wein, R., Fogel, E., Zukerman, B., and Halperin, D. 2007. Advanced programming techniques applied to cgal's arrangement package. Comput. Geom. Theory Appl. 38, 1-2, 3763.

YAP, C. K. 1997. Towards exact geometric computation. Comput. Geom. Theory Appl. 7. 